\title{
GWAgeER - A GroupWise Age Ranking Framework for Human Age Estimation
}

\author{
Olufade F. W. Onifade, Damilola J. Akinyemi \\ Department of Computer Science, University of Ibadan, Ibadan, 20001, Nigeria \\ E-mail: olufadeo@gmail.com, akinyemijd@gmail.com
}

\begin{abstract}
The task of estimating the age of humans from facial image is a challenging one due to the non-linear and personalized pattern of aging differing from one individual to another. In this work, we investigated the problem of estimating the age of humans from their facial image using a GroupWise age ranking approach complemented by ageing pattern correlation learning. In our proposed GroupWise age-ranking approach, we constructed a reference image set grouped according to ages for each individual in the reference set and used this to obtain age-ranks for each age group in the reference set. The constructed reference set was used to obtain transformed LBP features called age-rank-biased LBP (arLBP) features which were used with attached ageranks to train an age estimating function for predicting the ages of test images. Our experiments on the publicly available FG-NET dataset and a locally collected dataset (FAGE) shows the best known age estimation accuracy with MAE of 2.34 years on FG-NET using the leave-oneperson-out strategy.
\end{abstract}

Index Terms - Age estimation, age rank, age-rank-bias, groupwise age-ranking, ranking.

\section{INTRODUCTION}

The phenomenon of human ageing is a complicated aspect of human physiology that has been studied in various disciplines such as Human Perception and Psychophysics [1], Computer Vision and Image Processing [2], Pattern Recognition [3] and Forensic Science [4]. Human ageing is particularly complicated due to the several factors that influence it as well as the fact that it is temporal (time-variant) and personalized. Humans age in different ways depending on several factors which range from external factors such as weather, condition of living, drugs, nutrition etc to internal factors such as gender, heredity, ethnicity etc. As complicated as human ageing is, humans seem to be able to predict the ages of other humans relatively accurately with little effort [5] and this is done using heuristics. However, this task is not as easy for machines as it is for humans

In Computer Vision, Image processing and Pattern Recognition research, age estimation is considered as the task of predicting or determining the age or age range of an individual given certain parameters (often, the face) [6]. Interestingly, age estimation has received a considerable amount of attention in the research community owing to its wide range of applicability which includes a number of adaptive technologies such AgeSpecific Human Computer Interaction (ASHCI) [7], age falsification detection in sports and military [8] and even Security and Surveillance [9].

However, the complicated nature of this task, as explained earlier, has given rise to several different approaches to estimating the ages of humans to a reliable degree of accuracy. In this paper, we propose an age estimation approach called GroupWise Age Estimation by Ranking (GWAgeER). Our novel approach employs GroupWise age-ranking to determine the ages of subjects from their facial images and in the process, learns the correlation between the various ageing patterns of different individuals within each age group. Thus, we were able to come up with an age estimation framework that intuitively combines ageing pattern learning and age ranking for determining ages; to the best of our knowledge, this is the first research in age estimation to employ this approach. Our results indicate significant improvement over the state-of-the-art approaches.

The remainder of this paper is organized as follows; section II contains a review of previous literature on age estimation research, section III discusses the methodology of the proposed age estimation framework, section IV gives details of our experiments and section V gives the results obtained from them with a comprehensive comparison with the results from existing works and the conclusion is presented in section VI of this write up.

\section{RELATED WORKS}

Over the years, research in human age estimation has achieved significant results, which seem to have outperformed human age estimation ability. However, the need to obtain more reliable age estimates which are close to ground truth age has caused age estimation research to keep improving. So far, the best reported results on the two most widely used facial ageing datasets FG-NET [10] and MORPH [11] obtained Mean Absolute Error (MAE) of 3.14 years and 4.18 years on FG-NET and MORPH respectively $[12,13]$. From our observation, existing research in age estimation can be categorized into five major categories based on the approach employed for determining age [8]. These categories are 
the Anthropometric models, the Ageing Pattern Learning models, the Multiclass approach, Regression approach, the Age Ranking approach. Most other approaches are hybrid of two or more of the above stated.

Anthropometry, the science of measuring sizes and proportions of objects, has been used both to model the progression of growth of human faces as well as to estimate the ages of humans based on their faces [14]. The Anthropometric Models [2, 15, 16] adopt knowledge from Facial Wrinkle Analysis and Craniofacial Research for modelling the growth of the face. They basically used age-related facial contour and texture changes to categorize faces into different age groups. This approach is therefore mostly suitable for young faces in which facial contour changes are still observable but is not quite suitable for older faces in which facial contour changes are not pronounced.

The Ageing Pattern Learning models are been based on the observation that individuals possess certain personalized ageing pattern, which is responsible for their ageing process $[9,17,18,19]$. These individual ageing patterns are therefore learnt in order to synthesize a facial image for individuals at some other ages not present in the training sample. Although, age estimation algorithms based on this approach were relatively successful, ageing pattern learning works best with images properly represented (in terms of gender, ethnicity and ageing pattern) in the training set and it requires complete face/age information about each subject to perform well. Unfortunately, there is currently no known facial ageing dataset that has as much information as is required for accurate ageing pattern learning [6]; FG-NET contains a wide range of age-separated facial images per subject but contains images for only one ethnicity (Caucasian) while MORPH is a multi-ethnic dataset but has a limited number of age separated images per subject (averagely, four images per subject) with age difference between such age-separated images less than one year.

The Multi-class approach [5, 20, 21] assumes that age labels are independent classes into which face images can be classified thus resulting in a 'multi-class' classification problem. This approach has also been successful in age determination, especially due to the use of the Support Vector Machine (SVM) [22, 23]; an excellent Machine learning algorithm for classification. Unfortunately, the assumption that ages are independent is not quite realistic as close age labels often reflect similar ageing features; this therefore, has limited the performance of algorithms based on this approach.

Age estimation has also been handled as a regression problem [7, 13, 24, 25, 26] in which the age labels are learnt by a function which fits face images to their corresponding ages in an attempt to estimate their ages. This is intuitive because the age labels are integers and their relationship with the ageing features, expressed as real numbers, can be learnt. Support Vector Regression (SVR) has been very successful in this approach, thus researchers have applied several modifications of it to improve the model fitting function. This approach often requires a relatively large database of ageing faces and rigorous training.

The ranking approach regards age labels as ordinal pairs and the age of a test image is estimated based on some preferences obtained by comparing the rank of this image with the rank of a set of reference images whose ages are known [27, 28, 29, 30 31, 32]. This approach has met with a level of success surpassing that of the classification and regression approaches because of its intuitive method of determining an unknown age of a facial image by comparison with facial images of known ages to determine whether it is older or younger and eventually using this preference to arrive at an age estimate.

Of all the five categories, the ranking approach seem to be the closest to the way humans estimate ages; i.e. comparing the face of the subject in question across a set of other faces whose ages are known and trying to see where the face in question should fit in the set. In this work, we employ the ranking approach to age estimation, but with intuitive improvements. Drawing inference from the listwise ranking approach of Information Retrieval $[33,34,35]$, the proposed age estimation framework ranks facial images by performing a group-wise comparison of facial images instead of the pairwise comparison employed in previous ranking frameworks.

\section{PROPOSED ALgORITHM}

Recent research have employed the Ranking Approach to Age Estimation [28, 29, 30, 31] on the basis that it is intuitively easier to predict the older or younger between two or more faces than it is to predict the age of a particular face, therefore stating that age estimation is easier by establishing relationships or correlation between faces. These works employed a reference set of ranked images organized as a series of pair-wise images such that a test image is compared against each pair in order to determine the rank of the test image. The pairs were organized such that one image is older than the other in every pair, thus they used the relationship between facial images of different ages and the pairs were called ordinal pairs. Ref. [28] improved on the pairwise organisation of images used in previous approaches by including what they called "consistent pairs" which contained pairs of images from different subjects of the same age. By so doing, they included in the reference set, information about the relationship between similarly aged faces; thus, their reference set contained both ordinal and consistent pairs. To estimate the age of a test image, a series of pairwise comparisons was carried out along both the ordinal and consistent pairs to determine the rank of such test image before its age is estimated.

From the description of the above ranking approaches employed for age estimation, our observation is that there is a limit placed on the age learning function to use only the information available from pairs of images (due to the pair-wise organization of images in the reference set), not being able to learn certain informative trends in the ageing pattern of different individuals. Also, as observed 
in [3, 29, 31], ages have some relationship with other ages around or close to them, that is, a facial image labeled as 17 years may also reflect features similar to that of facial images that are 16 and 18 years old for instance. This therefore indicates that using ranks for exact ages, a person of age 17 could be ranked as either older or younger than his actual age (17); this could have negative impacts on the result of age estimation.

To combat this, our work proposed a group-wise organization of the image reference set so that images are organised in groups according to individual ageing patterns. In other words, several age-separated images of different individuals are clustered according to age groups instead of exact ages. Therefore, our reference set can be simply viewed as a matrix of images in which images in each column represent different age-separated images of an individual and images in a row are images of different individuals in the same age group. This presents two advantages; first the reference provides sufficient information about individual ageing patterns as well as the correlation/relationships between them across different individuals, secondly, it reduces the possibility of incorrect ranking of images whose ages lie close to one another since ranks are related to age groups rather than exact ages.

We also observed that most existing works employing the ranking approach to age estimation, except [30], do not give appropriate consideration to feature dimensionality reduction. In the proposed GroupWise ranking approach, dimensionality reduction is achieved with the age-rank-biased texture features. Using the GroupWise age ranking framework, we were able to transform facial texture features to reflect age-relevant texture features while reducing the dimensionality of the texture features as much as possible. To the best of our knowledge, this is the first research in age estimation to employ a "non-pairwise" ranking approach for age ranking.

Fig. 1 is a generic model showing the overview of our proposed age estimation framework. As indicated in the figure, the age estimation process begins by preprocessing images after which texture features are extracted from each facial image using the Local Binary Pattern (LBP) operator [36]. The proposed GroupWise age ranking approach is used to transform the obtained texture features in order to reflect ageing; this is shown by the bi-directional arrow between the two modules age-rank-biased features transformation. Consequently, the age-rank-biased texture features of an input image along with its age rank (as computed by the GW ageranker) are used to determine the age of such input image.

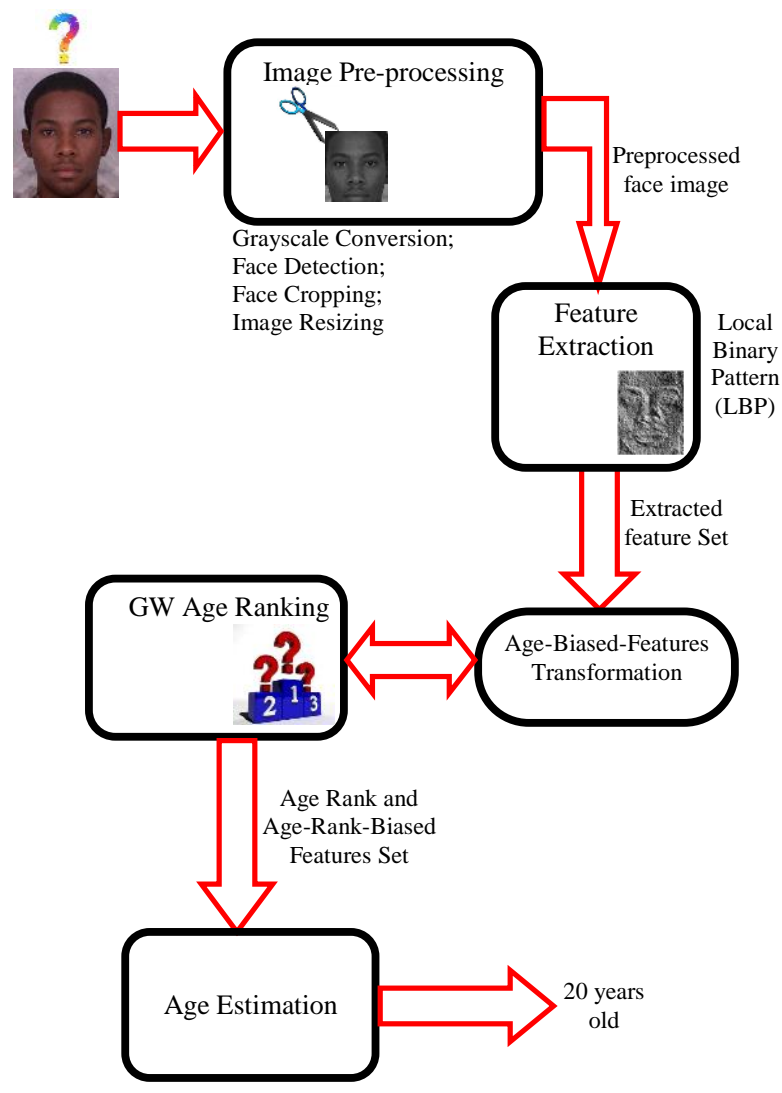

Fig.1 A generic model of the proposed age estimation Framework

\section{A. GW Age Ranking}

As earlier stated, the proposed GroupWise age-ranking framework takes inference from the listwise ranking approach of Information Retrieval. However, whilst the listwise approach ranks the relevance of documents to a query by taking a list of documents as an instance, our proposed approach does not only take the group of images as an instance, but in the course of ranking, it considers the individual images in the group and their relationships with one another in order to determine a rank for images in the age group. Therefore, the rank of each age group reflects the generic characteristics of the group as well as the relationships between characteristics of individual images in the age group. We present below, the Mathematical formulations for the proposed GroupWise Age Ranking approach.

Definition 1: Given a list of objects (facial images), $X$ and an outcome space $Y$ (of age labels), we state the following definitions.

$$
\begin{gathered}
X=\left\{x_{1}, x_{2}, \ldots, x_{n}\right\} \\
Y=\left\{y_{1}, y_{2}, \ldots, y_{m} \mid \forall i y_{i}>y_{i-1}\right\}
\end{gathered}
$$

So that each image $x_{i} \epsilon X$ has an age label $y_{i} \epsilon Y$ and this relationship is many-to-one and onto i.e. many images can have the same age label but no single image can have more than one label. This relationship can be expressed 
as follows;

$$
\left(x_{i}, x_{i+1}, \ldots\right) \rightarrow y_{j} ; x_{i} \mapsto\left(y_{j}, y_{j+1}, \ldots\right)
$$

The symbol $\mapsto$ indicates that there no such mapping exists between the two operands. Therefore, from (1) and (2), we can say that, $m \leq n$.

Suppose we can define a particular subset of $X$ as $X_{i} \subset X$, such that $X_{i}$ contains objects (images) belonging to a particular age group specified by an age range, $\rho$. We therefore wish to define $k$ such subsets of $X$ as follows.

$$
X_{1} \cap X_{2} \cap \ldots \cap X_{k}=\{\} ; \forall X_{j} \subset X
$$

From (4), each $X_{j}$ is a distinct subset of $\mathrm{X}$ and thus each $x_{i} \in X$ belongs to exactly one $X_{j}$. Therefore, we can state the following equation

$$
\forall x_{i} \exists X_{j} x_{i} \in X_{j} ; i=1,2, \ldots, n ; j=1,2, \ldots, k
$$

Each image age group, $X_{j}$, consists of $f$ different individuals so that each $X_{j}$ can be written as

$$
X_{j}=\left\{x_{j 1}, x_{j 2}, \ldots, x_{j f}\right\}
$$

Note that, $f$ being the number of individuals in each age group, is required to be consistent all through the age groups (i.e. each individual is represented once in each age group). In our experiments, we set $f=12$.

Therefore our reference set can be viewed as a matrix, $\theta$, written as

$$
\theta=\left[\begin{array}{cccc}
X_{11} & X_{12} & \cdots & X_{1 f} \\
X_{21} & X_{22} & \cdots & X_{2 f} \\
\vdots & \vdots & \vdots & \vdots \\
X_{k 1} & X_{k 2} & \cdots & X_{k f}
\end{array}\right]
$$

Note that, $x_{i}$ as defined in (1) is now equivalent to $X_{i j}$

Consequently, $Y$ can be partitioned into $k$ disjoint subsets according to the age range, $\rho$, as follows:

$$
Y_{1} \cap Y_{2} \cap \ldots \cap Y_{k}=\{\} ; \forall Y_{j} \subset X \mid c\left(Y_{j}\right)=\rho
$$

such that each image $x_{i} \epsilon X_{j}$ maps to a corresponding age label in $Y_{j}$ and $c($.$) is a function that returns the cardinality$ of its argument (a set).

We can therefore write each $Y_{j}$ as a vector, $\phi$

$$
\phi=\left[\begin{array}{c}
Y_{1} \\
Y_{2} \\
\vdots \\
Y_{k}
\end{array}\right]
$$

Definition 2: Suppose there is an arbitrary function agegroup(.) which maps each subset of $X$ to its corresponding subset in $Y$, then we can write

$$
\forall X_{j} \exists Y_{j} \operatorname{agegroup}\left(X_{j}\right) \rightarrow j ; \forall j=1,2, \ldots k
$$

Definition 3: We remember that $Y$ is the outcome space (of age labels) and that each $Y_{j} \subset Y$, contains age labels in $Y$ that belong to the same age group and are therefore assumed to have the same (age-group) rank.

Therefore, we can define an age-group-specific ranking function given a set $\mathrm{R}$ of ranks. For now, we will abstract our ranking function as rank (.)

$$
\begin{gathered}
R=\left\{r_{1}, r_{2}, \ldots, r_{k} \mid r_{j} \in \mathfrak{R}\right\} \\
\forall Y_{j} \exists r_{j} \operatorname{rank}\left(Y_{j}\right) \rightarrow r_{j} ; \forall j=1,2, \ldots k
\end{gathered}
$$

Thus, from (10), we can map each subset of $X$ (i.e. $X_{j} \subset X$ ) to a rank $r_{j} \in R$ as follows:

$$
\forall X_{j} \exists r_{j} \operatorname{rank}\left(X_{j}\right) \rightarrow r_{j} ; \forall j=1,2, \ldots k
$$

Therefore, each image in each $X_{j}$, as defined in (5) has exactly one $r_{j}$ according to (12) and (13)

$$
\forall X_{j} \exists r_{j}\left(\operatorname{rank}\left(x_{j}\right) \rightarrow r_{j}\right) ; x_{i} \in X_{j}
$$

Where $\forall i=1,2, \ldots, n j=1,2, \ldots k$.

So far, we have used $\operatorname{rank}($.$) as an abstraction of our$ ranking function, we now provide adequate definitions for our proposed groupwise ranking function.

Definition 4: Suppose we have a space $H$ of ranking functions defined as follows

$$
H=\{h(.)\}
$$

Thus, we can redefine (14) as

$$
h\left(x_{i}\right) \rightarrow r_{j}
$$

Where

$$
\forall x_{i} \in X_{j} \& r_{j} \in R ; i=1,2, \ldots, n ; j=1,2, \ldots, k
$$

Our goal is to find an $h($.$) with the least error/loss on$ ranking so that estimation error can be reduced to the barest minimum. The error on ranking has a direct effect on the amount of deviation from the ground truth age during age estimation; therefore minimizing the error on ranking could reduce the likely amount of deviation during age estimation. Thus, we define the loss/error on ranking as $\mathcal{E}_{r}$. 


$$
\varepsilon_{r}=\sum\left(\left|\bar{r}_{j}-r_{j}\right|\right)^{2} / N
$$

Where,

$$
\begin{aligned}
& \quad \bar{r}_{j}=\text { Predicted rank (expected to lie close to the actual } \\
& \text { rank values) } \\
& \quad r_{j}=\text { actual rank in } R \\
& N=\text { Number of observations }
\end{aligned}
$$

Specifically, $h($.$) must learn to rank images in each X_{j}$ in a groupwise manner by relating the texture features of each image in an age group to the median age of the age group. However, the texture features could describe various characteristics of the human face and there is need to ensure that only age-relevant texture features are considered for age ranking; this is what age-rank-biased Feature transformation helps achieve. Age-rank-biased feature transformation is explained in the next section.

The model for the proposed GW Age-Ranking is shown in Fig. 2. As shown in the figure, the images in the reference set are arranged to reflect the ageing pattern of different individuals across different age groups. Also, unlike existing age ranking techniques, the $\mathrm{GW}$ age ranking approach produces age-group ranks which give inferences into the age group to which a particular facial image belongs so that these inferences can be utilized to estimate the ages of individuals. Images surrounded with dashed lines are images of individuals used as substitutes where images of particular individuals are missing for those age groups.

\section{B. Age-Rank-Biased Features Transformation}

As shown in the methodology in Fig. 1, facial texture features are obtained using Local Binary Patterns (LBP). LBP is an efficient texture operator that has been applied in several Pattern Recognition problems with good performance. LBP is particularly useful for describing image texture in terms of pixels (threshold and neighbourhood pixels) which are codified into binary values. In the case of facial images, it helps describes the facial skin texture and this is a feature of interest in this research. However, the face (even facial texture) describes a number of facial characteristics apart from age; facial expression and recognition features could also be obtained from the face. In a bid to ensure that the facial features reflect ageing (especially, ageing pattern), there is a need to locate and explore age-relevant texture features. To this end, we employed age-rank-biased LBP, a method that achieves the stated goal by transforming texture features such that they reflect ageing patterns and reduces the dimensionality of the obtained texture features.

Using the GW age-ranking model and the constructed image reference set, the age-rank-biased feature set is obtained as an age-rank-biased LBP features (LBP). Basically, arLBP is obtained by carrying out a set of computations first on the texture features of images in the reference set and then on the texture features of input images. For the texture features of reference images, the computed age-ranks in (16) are used to bias features by taking a groupwise product of the features and the agegroup ranks and squaring the result. However, for input images, a weighted dissimilarity computation is carried out between the arLBp features of the reference image and the texture features of the input image. The weighted dissimilarity measurement is taken across each age group taking into cognizance specific features obtained from specific regions of the face (as specified in (11)) from each individual in each age group. By so doing, the obtained features for an input image is a reflection of the relationship between the features of different individuals in an age-group, thus enabling the learning of individual ageing patterns across the different age groups available, since the age-separated images of individuals in the reference set are arranged to reflect ageing patterns across different individuals as shown in Fig. 2.

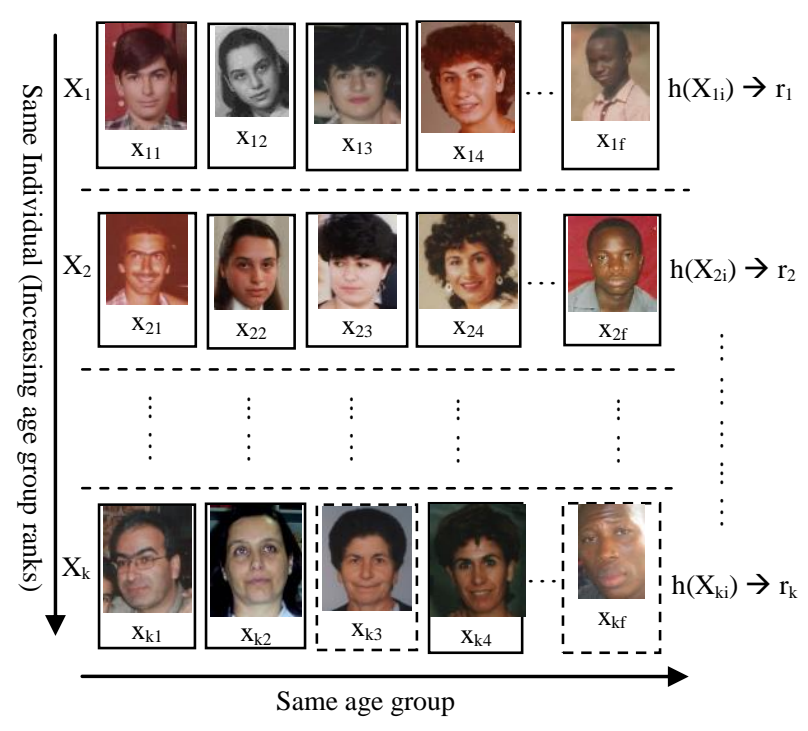

Fig. 2 Proposed GW age-ranking model

Mathematically, arLBP is modeled as follows:

Definition 5: Given a reference image set containing age-grouped images $X_{1}, X_{2}, \ldots, X_{n}$, with $b_{i}$ being the histogram of LBP features of each image in each $X_{j}$; each $X_{j}$ is then biased with the age rank $\left(r_{j}\right)$ of its age group as follows:

$$
\begin{gathered}
b_{i} \leftarrow L B P\left(x_{i}\right) ; \forall x_{i} \in X_{j}, b_{i} \in \beta_{j} \\
r_{i} \cdot{ }^{*} \beta_{j}=\beta_{j}^{\tau}
\end{gathered}
$$

Where

$r_{j}=$ agerank of $X_{j}$ (obtained according to (16))

* is an element-wise matrix product operator.

$\beta_{j}=$ Histogram of LBP features of all images $x_{i}$ in $X_{j}$

$\beta_{j}^{\tau}=$ agerankbiased LBP features of all images $x_{i}$ in $X_{j}$

$L B P$ (.) is a function that computes the Local Binary Pattern of an image.

Definition 6: Given an image $x_{i}$ with LBP histogram $b_{i}$ and a reference image set containing age-grouped images 
$X_{1}, X_{2}, \ldots, X_{n}$; arLBP of image $x_{i}$ can be computed as follows

$$
b_{i} \bullet \beta_{j}^{\tau}=b_{i}^{\tau} ; b_{i}^{\tau}=\left\{b_{i}^{\tau} \mid i=1,2, \ldots, k\right\}
$$

Where

$$
\forall j=1,2, \ldots k
$$

$k=$ the number of age groups used in the reference set

- is a weighted-accumulated difference operator.

$b_{i}^{\tau}=$ the resulting Age-Rank-Biased feature set for $x$.

- is an operator that performs a weighted accumulated difference across different age groups in the reference set and implemented as shown in Fig. 3.

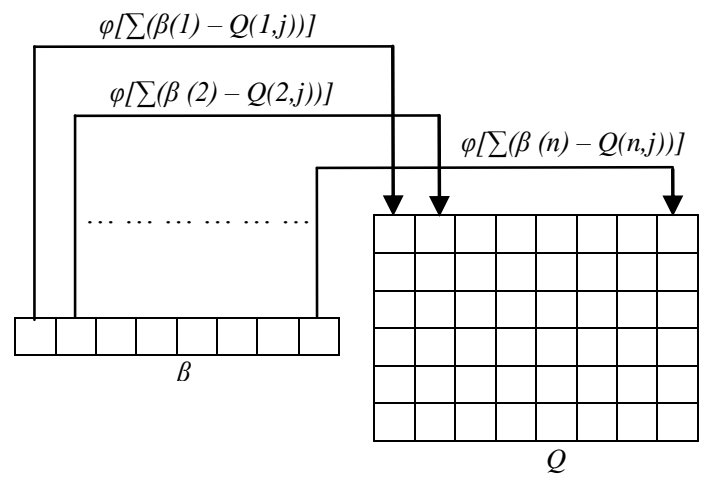

Fig. 3 Histogram dissimilarity computation for arLBP

In Fig. 3, $\beta$ represents the histogram of features of the input image while $Q$ represents those of the reference images; $\varphi$ is a function that retrieves the weights of respective facial regions. The difference in square brackets is the difference between corresponding features of the input image and the reference images; this difference is multiplied with their respective weights (obtained by $\varphi$ ) and is accumulated for each feature per age group in the reference set. Thus, the result is an accumulation of weighted differences between the features of the input image and those of the images each age group in the reference image set.

AR LBP is actually a bias of the histogram of LBP features. It uses the age ranks of images in the reference set to bias the pattern and distribution of image texture features obtained thereby reflecting the relationship between the image texture features and their age ranks (age groups). Subsequently, these age-rank-biased features are used to produce more than one feature vector for each input image thus producing more observations for training.

In other to keep this paper focused on the important contributions of this work, it does not include detailed discussion of other concepts which were part of the work but were not major contributions of the work; an example of this is the Local Binary Pattern (LBP) which is the technique used for describing the facial texture transformed into arLBP. Details of LBP and its various implementations can be found in $[36,37,38]$. Implementation-specific details of the GW age-ranking and arLBP models are explained in the next section.

The following algorithms were for constructing the image reference set and for ranking images respectively using the proposed GW age-ranking approach.

1. Input: $X=\left\{x_{1}, x_{2}, \ldots x_{n}\right\}$ (a set of images), and $Y=$ $\left\{y_{1}, y_{2}, \ldots, y_{n} \mid y_{i} \leq y_{i+1}\right\}$ (a set of age labels) where $m \leq n$.

2. Construct reference set, $\theta$ :

a. Define age range $=\rho$; number of age groups in $\theta$ $=k$; number of individuals per age group in $\theta=f$.

b. Construct each age group $X_{j} \subset X$ according to (4) and (5).

c. Construct $Y_{j} \subset Y$ according to the equation in (8) and label each $x_{i} \in X_{j}$ with its corresponding age group label in $\phi$ from (9). Thus, $Y_{j}$ becomes the age group label for $X_{j} ; Y_{j+1}$ the age group for $X_{j+1}$ and so on.

d. Construct the reference set, $\theta$ attached to the age group labels in $\phi$.

$$
\theta=\left[\begin{array}{cccc}
X_{11} & X_{12} & \cdots & X_{1 f} \\
X_{21} & X_{22} & \cdots & X_{2 f} \\
\vdots & \vdots & \vdots & \vdots \\
X_{k 1} & X_{k 2} & \cdots & X_{k f}
\end{array}\right] \phi=\left[\begin{array}{c}
Y_{1} \\
Y_{2} \\
\vdots \\
Y_{k}
\end{array}\right]
$$

3. Feature Extraction: Extract LBP features for all images in $\theta$ and compute the histogram of their LBP features.

for each $X_{i j}$ in $\theta$

end

$$
\beta_{i j} \leftarrow \operatorname{LBP}\left(X_{i j}\right)
$$

4. Age Rank Computation: In each age group, learn a common trend and use it to determine their age ranks.

for each $X_{i}$ (age group) in $\theta$

for each $X_{i j}$ in $X_{i}$

end

$$
h\left(X_{i j}\right) \rightarrow r_{i j}
$$

$r_{i} \leftarrow \operatorname{learn}\left(r_{i j}\right)$

end

5. Calculate the age-bias for each age group in $\theta$ : (From step 3, each $X_{i j}$ in $\theta$ has histogram features $\beta_{i j}$ )

for each $X_{i}$ (age group) in $\theta$ for each $X_{i j}$ in $X_{i}$

$$
b_{i j}^{\tau} \leftarrow \operatorname{AgeRankBias}\left(r_{i}, \beta_{i j}\right)
$$

end

end

Algorithm. 1 ConstructGWRank $(\bullet)$ Algorithm for constructing the groupwise reference image set 
6. Output: $\theta=\left[\begin{array}{cccc}X_{11} & X_{12} & \cdots & X_{1 f} \\ X_{21} & X_{22} & \cdots & X_{2 f} \\ \vdots & \vdots & \vdots & \vdots \\ X_{k 1} & X_{k 2} & \cdots & X_{k f}\end{array}\right] \phi=\left[\begin{array}{c}Y_{1} \\ Y_{2} \\ \vdots \\ Y_{k}\end{array}\right]$

The learn() function of step 4 in Algorithm 1 is an implementation of the way the ranking function $h($.$) was$ learnt.

1. Input: histogram features $H_{x}$ for a given image $x$

2. Compute histogram dissimilarity: (From step 5 in Algorithm 1, the age-biased feature of each image in the reference set, $\theta$, is denoted $b_{i j}^{\tau}$ )

for each $X_{i}$ (age group) in $\theta$

for each $X_{i j}$ in $X_{i}$

$$
b_{x i}^{\tau} \leftarrow \operatorname{AgeRankBias}\left(\beta_{i j}, b_{i j}^{\tau}\right)
$$

end

$$
b_{x}^{\tau} \leftarrow b_{x i}^{\tau}
$$

end

3. Output: $b_{x}^{\tau}=\left\{b_{x 1}^{\tau}, b_{x 2}^{\tau}, \ldots, b_{x k}^{\tau}\right\}$ where each $b_{x i}^{\tau}$ is a vector of age-biased texture features.

Algorithm. 2 GWRanker $(\bullet)$ : Algorithm for ranking an input image.

\section{EXPERIMENTS}

In the experiments, we employed a standard facial ageing dataset (FG-NET [10]) along with a locally collected dataset (FAGE). The FG-NET dataset is a standard facial ageing dataset of 1002 facial images of 82 Caucasian unique subjects with an average of 12 ageseparated facial images per subject. The FAGE dataset is a locally collected facial ageing dataset of 115 facial images of 87 indigenous African subjects (as at the time of this experiment, work is still on to increase the size of the dataset). Although, the FAGE dataset is relatively small in size, it is peculiar to this research because it contains faces of indigenous African - faces of African who reside within the African continent. To the best of our knowledge, this is the first facial ageing dataset to feature indigenous African faces, existing facial ageing datasets such as MORPH [11] (which is about the largest known multi-ethnic facial ageing dataset) contain facial images of Africans residing outside the continent; based on the observation of the impact of weather and condition of living on ageing [7, 39], we believe that this feature of indigenous faces is equally impactful on the result of age estimation. Consequently, we believe this is the first age estimation research on indigenous African faces.

Our experiments were focused on estimating the ages of individuals within the teenage and young adult period of human life (13 - 40 years). We chose this age range based on our observation that this is the period of human life with the most active involvement in social activities and is therefore the most crime-prone period of life; research is still on to evaluate the performance of the proposed methodology on a wider age range. Basically, all our experiments were performed using a combination of the two aforementioned datasets with MATLAB ${ }^{\circledR}$ software for implementation, but evaluations were carried out on the dataset both separately and as a combination.

Following the proposed GW age ranking model, an image reference set was constructed from the two aforementioned datasets using a subset of the combination of both datasets. Our reference set consisted of 12 unique subjects with 7 age-separated facial images per subject to represent 7 different age groups $(13-16$; 17 - 20; 21 - 24; 25 - 28; 29 - 32; 33 - 36; 37 - 40). For subjects with missing facial images for a particular age group, other subjects were used as substitutes (these are indicated in Fig. 2 as images surrounded with dashed lines). Facial texture features of images were extracted using Local Binary Patterns and the distribution of the texture features were observed by obtaining the histogram of these features. Specifically, the $h($.$) ranking$ function was implemented as follows:

I. First, an empirical study of the distribution of facial texture features was carried out by observing the standard deviation of the features of each individual in each age group and comparing it with the standard deviation of facial texture features of all individuals in each age group.

a. It was observed that more than $70 \%$ of images in each age group in the reference set had the standard deviation of their features close to that of the entire age group with a difference of less than 2 .

b. It was also observed that the standard deviation of each age group in the reference set decreased from the smaller age to the higher ones. The only exception to this was the second to the last age range (33 - 36 years) whose standard deviation suddenly shut up, yet the last age range $(37-40$ years) still had a lower standard deviation value than this second to the last age range ( $33-36$ years). However, this last two age ranges had standard deviation values higher than the third to the last age range (29- 32 years) and the effect of this deviation was revealed on the results as presented in section $\mathrm{V}$. This discrepancy is due to the sparse distribution of images in this age range as well as the limited number of images in this age range; there is a sharp difference in the number of images in the age range 29 - 32 years and the age range $33-36$ years (49 and 39 images).

II. Age ranks were computed for each age group using the formula;

$$
r_{j}=\operatorname{std}\left(X_{j}\right) \times \operatorname{mean}\left(Y_{j}\right)
$$

Where $\operatorname{std}($.$) gives the standard deviation of the image$ features and mean(.) gives the mean of the age labels in each age group and all other variables remain as previously defined. 
III. Subsequently, the age ranks of each age group were used to bias the texture features of images in the age group by factoring the images in the age group by the age group rank along individual features of images in each age group.

IV. The obtained standard deviation of each age group in (I) was combined with the image features in different ways as shown in Table 1 to arrive at different age ranking parameters for each age group.

V. arLBP was employed according to (20) and Fig. 3 to obtain age-relevant texture features for every image in the training set by ranking input images based on their relationship with each age group; thus obtaining a set of features per image which reflects the relationship of this image across different age groups.

VI. An ensemble learning framework was employed with a regression method to learn these various age ranking parameters with the facial image features and the rank parameter with the least error on ranking is eventually employed for training and ranking facial images.

Subsequently, the goal of the proposed GW ageranking model is achieved by obtaining the ranking parameter with the least error evaluated using (17). As seen from Table 1, the type 1 ranking parameter (i.e. the age ranking parameter obtained according to (21)) obtains an error level lower than the age range of its rank values and this was the lowest among the three 'types'; thus, type 1 ranking parameter was employed throughout the experiments. In our experiments, $h($.$) was$ implemented with LSBoost [40], a boosted regression method for ensemble learning. Hence, the groupwise agerank learning of $h($.$) is particularly aided by arLBP as in$ step (V). Our choice of LSBoost for age ranking is because it fits regression ensembles by minimizing the least squares error on prediction and this quantity (least squares error) is close to the mean squared error used for evaluating the error on ranking specified in (20). LSBoost was also used as the age estimation function due to its regression ability. For both age ranking and estimation, we used the ensemble learning framework of MATLAB ${ }^{\circledR}$ using a regression tree template with pruning and LSBoost as the only weak learner in the ensemble. For validated trainings, we used a learning rate of 0.1 for validation protocols and 1 for independent test sets; lower learning rates generally gives more accuracy on cross validated ensembles but this is not always the case with predictions on out-of-training samples.

For the estimation of ages from facial images, the learnt ranks were used with image features to determine the ages of facial images. Specifically, the texture features of facial images were transformed into agerelevant texture features using our arLBP approach and the age-relevant texture features are eventually used for age estimation. Therefore, following from (19), Fig. 3 and Algorithm 2, arLBP eventually represents every input image with 7 different vectors of age-relevant texture features; each feature vector representing the relationship of an input image with each age group in the reference set. In this way, we were able to capture the relationship of an input image across every age group and were therefore able to represent its age-relevant texture features to reflect the characteristics of the GW age-ranked reference set - namely the ageing pattern variations and the GroupWise age ranking. This presents two advantages; first it provides several different feature vectors for determining the age rank and age estimate of a single image thus reducing the possibility of wide deviation from the ground truth age during age estimation. Secondly, it systematically increases the size of the training set and this helps complement the small size of our FAGE dataset, because the learning algorithm is able to learn with more observations.

The obtained set of features vectors were then trained for ranking using LSBoost and the obtained ranks together with these features were used for age estimation. In order to appropriately justify the performance of the proposed approach, our experiments were carried out under various validation settings.

Table. 1 Age Ranking Parameters

\begin{tabular}{|c|l|l|l|}
\hline $\begin{array}{c}\text { Type of } \\
\text { Rank } \\
\text { used }\end{array}$ & \multicolumn{1}{|c|}{$\begin{array}{c}\text { Minimum \& } \\
\text { Maximum values }\end{array}$} & $\begin{array}{c}\text { Range of } \\
\text { Values }\end{array}$ & $\begin{array}{c}\text { Loss on } \\
\text { Ranking }\end{array}$ \\
\hline Type $1^{\mathrm{a}}$ & $0.0067,0.0588$ & 0.0074 & 0.0000145 \\
\hline Type 2 & $147.3475,176.8391$ & 4.2129 & 6.4688 \\
\hline Type $3^{\mathrm{c}}$ & $18725.79,106931.73$ & 12600.85 & 13632564.1 \\
\hline
\end{tabular}
product of the standard deviation of each image and the mean of its respective age group

First, we validated our proposed approach using standard statistical cross validation protocols. However, based on the fact that some cross validation protocols overestimate the performance of learning algorithms [40], we also experimented the performance of our algorithm on an independent test which was explicitly or manually excluded from the training set. Although our approach was extensively tested on standard facial ageing datasets and not on live data, testing on an independent test set could serve to provide true and reliable estimates of the performance of the proposed algorithm for use in working systems where most input images that to be worked upon would be live images.

\section{RESUlTS AND DisCUSSION}

From previous research in age estimation, two standard evaluation metrics have been proposed for age estimation algorithms; namely Mean Absolute Error (MAE) and Cumulative Score (CS). MAE is the average/mean deviation of the estimated age from the ground truth (true) age while CS is the percentage of images for which estimation error falls below a particular threshold. The formulae for the metrics are given as: 


$$
\begin{gathered}
M A E=\sum(|\hat{y}-y|) / N \\
C S=\left(\sum(|\hat{y}-y|<\varepsilon) / N\right) \times 100 \%
\end{gathered}
$$

Where

$y=$ the ground truth (true) age; $\hat{y}=$ the machineestimated age; $\varepsilon=$ error threshold value; $N=$ number of observed image samples

Cross validation partition is a cross validation protocol which randomly splits the dataset into training and test set based on specified proportions. We specified a number of random splits as follows; $80 \%$ training images and $20 \%$ test images, $70 \%$ training images and $30 \%$ test images, $60 \%$ training images and $40 \%$ test images and $50 \%$ training images and $50 \%$ test images. The results as shown in Tables 2 and 3 indicate MAE of approximately 1 year for all partitioned cross validated trainings.

In evaluating the performance of our proposed method, other validation protocols used were the folded ( $\mathrm{k}$-fold) Cross Validation protocol. In a k-fold validation protocol the dataset is randomly split into $k$ different folds - where $k$ is a positive integer - each fold is then exempted from the dataset for testing while the remaining folds are used for training; this process is repeated until each fold has been used for once for training and $k-1$ times for training. Specifically, we used the popular leave-one-person-out (LOPO) protocol for validating on FG-NET. Similar to the k-fold validation protocol, LOPO leaves out the images of each subject in the FG-NET dataset once for testing and uses it $k-1$ times for training. In FG-NET dataset, $k=82$, however, considering the age range used in our experiments (13 - 40 years), three FG-NET subjects did not have any images in this age range, therefore, LOPO in this case has 79 subjects to deal with. As shown in Table 2, $\mathrm{k}$-fold cross validation was experimented using 4-fold, 5-fold and 10-fold cross validation and the results indicates that most of these validation protocols have MAE of approximately 2 years except in the case of LOPO with correct ranks (i.e. when age ranks were predicted fairly accurately).

Lastly, we explicitly excluded a carefully selected subset of the training set (17 images from FAGE and 54 images from FG-NET) such that each age range studied is represented in the subset; the subset is used as an independent test set as explained earlier. Altogether, 71 images were used for independent testing and to examine the effect of age ranking on the accuracy of age estimation, we experimented using correct and incorrect ranks. By correct ranks we mean a situation where images were ranked fairly accurately, and incorrect ranks refer to the situation in which age ranks were poorly predicted. From Table 2 and Table 3, age estimation results were best with ranks predicted accurately. All other cross validation settings for which the correctness of the ranks are not specified were experimented with incorrect ranks and this is an indication of an excellent performance of the proposed approach algorithm - even

Copyright $@ 2015$ MECS with incorrect ranks. It can therefore be said that GW age ranking is enhanced by the arLBP features when ranks are incorrect.

Table 3 gives details of the performance of the proposed age estimation framework on each age group used in the research. Although, the age ranks are predicted based on age groups, it is important to state that age estimation was done using exact ages unlike many existing works which classify facial images into age ranges. Due to the small age range covered in our experiments we have presented the MAE of Table 3 using a range of 3 years unlike the decade of life popularly used in FG-NET and MORPH datasets and we consider that this smaller age range presents a more detailed view of the performance of our age estimation framework on each age group. One can observe that the performance degraded with the higher ages $(29-40$ years) and this was due to the limited number of images available at those ages.

Fig. 4 and Fig. 5 give the Cumulative Scores (CS) on the in individual datasets as well as their combinations. It can be observed that Cumulative Scores for the k-fold validation protocols were not included in the graphs, this is because folded validations cannot be used for prediction on out-of-training samples; thus, evaluating the cumulative score would only be possible on the same data used for training which would not be appropriate. As seen in the figures, the cumulative scores were very high with correct ranks (Fig. 4) but with incorrect ranks (Fig. 5) a sharp difference is seen between the cross validation settings and the independent test set; an indication of the overestimation of cross validation protocols earlier stated.

To better evaluate the performance of our proposed age estimation framework, we presented in Table 4, detailed comparison of the performance of our approach (in terms of MAE) with the performance of state-of-the-art age estimation algorithms on the standard FG-NET dataset. Although, many of the compared works were tested on a wider age range $(0-69$ years $)$, it should be noted that a number of them classified images into age groups and not exact ages as is done in our approach.

Table. 2 MAE on the Combined Dataset (FAGE \& FG-NET)

\begin{tabular}{|l|c|}
\hline \multicolumn{1}{|c|}{ Training/Validation Configuration } & MAE ( years) \\
\hline CVPartition (80\%-20\%) & 0.9485 \\
\hline CVPartition (70\%-30\%) & 1.0282 \\
\hline CVPartition (60\%-40\%) & 1.1617 \\
\hline CVPartition (50\%-50\%) & 1.1876 \\
\hline 4-fold CV & 2.3761 \\
\hline 5-fold CV & 2.3214 \\
\hline 10-fold CV & 2.1915 \\
\hline LOPO (With incorrect ranks) & 2.3401 \\
\hline LOPO (With correct ranks) & 1.0034 \\
\hline Independent Test Set (correct ranks) & 1.7223 \\
\hline Independent Test Set (incorrect ranks) & 5.8511 \\
\hline
\end{tabular}

I.J. Image, Graphics and Signal Processing, 2015, 5, 1-12 
Table. 3 MAE on Each Dataset (FAGE \& FG-NET)

\begin{tabular}{|c|c|c|c|c|c|c|c|c|}
\hline $\begin{array}{c}\text { Age } \\
\text { Range } \\
\text { (years) }\end{array}$ & $\begin{array}{c}\mathbf{1 0} \text { F-fold } \\
\text { CV }\end{array}$ & $\begin{array}{c}\text { LOPO } \\
\text { (Incorrect } \\
\text { Ranks) }\end{array}$ & $\begin{array}{c}\text { LOPO } \\
\text { (Correct } \\
\text { Ranks) }\end{array}$ & $\begin{array}{c}\text { Independent } \\
\text { Test Set } \\
\text { (Incorrect } \\
\text { ranks) }\end{array}$ & $\begin{array}{c}\text { Independent } \\
\text { Test Set } \\
\text { (Correct } \\
\text { ranks) }\end{array}$ & $\mathbf{1 0 - f o l d ~ C V ~}$ & $\begin{array}{c}\text { Independent } \\
\text { Test Set } \\
\text { (Incorrect } \\
\text { ranks) }\end{array}$ & $\begin{array}{c}\text { Independent } \\
\text { Test Set/ } \\
\text { (Correct } \\
\text { ranks) }\end{array}$ \\
\hline $13-16$ & 2.1396 & 2.3879 & 0.8231 & 6.8571 & 1.2338 & 0.4339 & 6.0000 & 0.8571 \\
\hline $17-20$ & 1.7615 & 2.0182 & 1.0145 & 3.0000 & 1.5714 & 0.5397 & 3.2381 & 1.9524 \\
\hline $21-24$ & 1.8795 & 2.0022 & 1.3170 & 0.6939 & 1.8163 & 0.6818 & 2.0000 & 1.8286 \\
\hline $25-28$ & 2.1457 & 2.2241 & 1.1513 & 4.5893 & 1.4464 & 1.0095 & 2.3810 & 0.4286 \\
\hline $29-32$ & 1.8158 & 2.2105 & 0.8008 & 8.5714 & 2.3333 & 0.8701 & 6.5000 & 3.2143 \\
\hline $33-36$ & 2.2778 & 2.9206 & 1.0873 & 11.0408 & 2.8571 & 1.6500 & 13.000 & 2.4286 \\
\hline $37-40$ & 3.6149 & 4.2236 & 0.9689 & 14.6786 & 1.2143 & 0.7143 & 13.1429 & 1.4286 \\
\hline Overall & 2.0649 & 2.3401 & 1.0034 & 6.2487 & 1.7407 & 0.7024 & 4.5882 & 1.6639 \\
\hline
\end{tabular}

From Table 4, our approach significantly improves over the state-of-the-art age estimation approaches on FG-NET; even with incorrect ranks GWAGeER has MAE of 2.34 years. Also, as seen in the table, LOPO is the most widely accepted validation protocol on FG-NET and this is because of the wide range of age-separated images per subject present in the dataset. Fig. 6 also shows the Cumulative Score on FG-NET dataset from previous works. In the figure, GWAgeER1 refers to GWAgeER tested with LOPO (correct ranks) while GWAgeER2 refers to GWAGeER tested with LOPO (incorrect ranks). Obviously, GWAgeER1 has the best performance among the compared algorithms, achieving a cumulative score of $80 \%$ at error level 2 which is by far the best we have seen on FG-NET.

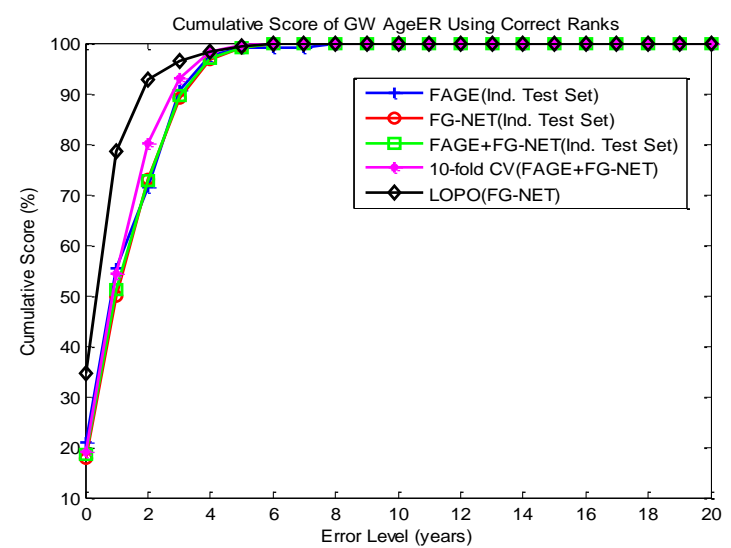

Fig. 4 CS on Correct ranks

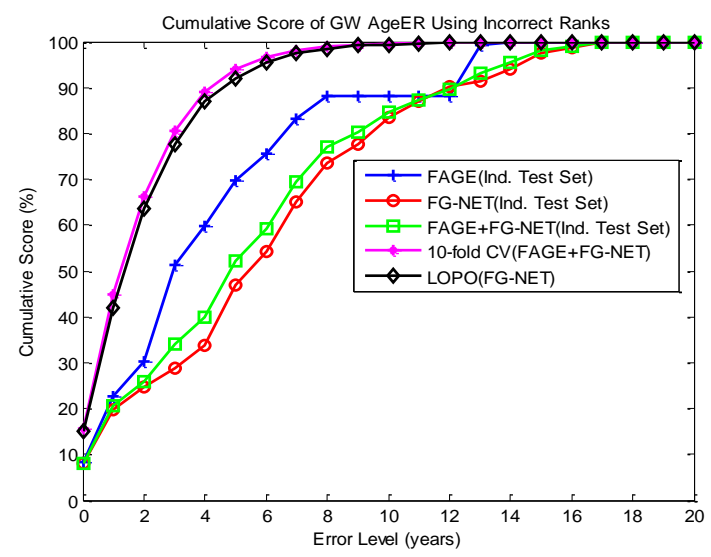

Fig. 5 CS on Incorrect Ranks

Copyright (C) 2015 MECS

\section{CONCLUSION}

This work has shown the impact of the ranking approach as well as the importance of employing agerelevant facial features to improving the age estimation. This work has contributed a novel and significant ranking approach which employs the correlation of ageing patterns of individuals across age groups as well as a method of transforming texture (LBP) features into agerelevant features which significantly improve age estimation.

Table. 4 Comparison of MAE on FG-NET

\begin{tabular}{|l|l|l|}
\hline \multicolumn{1}{|c|}{ Algorithm } & \multicolumn{1}{|c|}{ Validation Approach } & \multicolumn{1}{c|}{$\begin{array}{c}\text { MAE } \\
\text { (years) }\end{array}$} \\
\hline AGES $_{\text {lda }}[17]$ & LOPO & 6.22 \\
\hline RUN [27] & LOPO & 6.05 \\
\hline RPK [26] & LOPO & 4.95 \\
\hline BIF [5] & LOPO & 4.77 \\
\hline AAM+SVR [41] & Independent Test Set & 4.37 \\
\hline Rank [29] & (80-20) CVPartition & 5.79 \\
\hline EBIF [42] & LOPO & 3.17 \\
\hline RankBoost [30] & 4-fold & 5.67 \\
\hline OHRank [31] & (80-20) CVPartition & 4.48 \\
\hline C-lsLPP [3] & LOPO & 4.38 \\
\hline GWAgeER & LOPO (Incorrect ranks) & $\mathbf{2 . 3 4}$ \\
\hline GWAgeER & LOPO (Correct ranks) & $\mathbf{1 . 0 0}$ \\
\hline
\end{tabular}

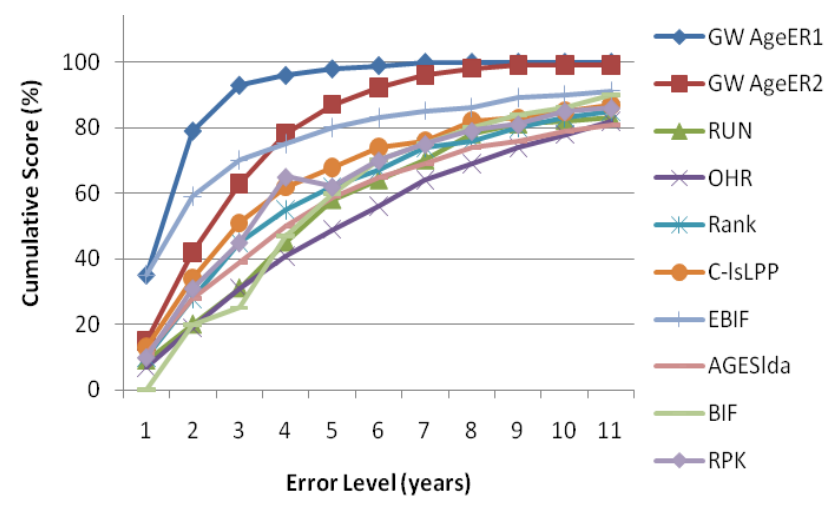

Fig. 6 Comparison of CS on FG-NET

Also, to the best of our knowledge, this is the first age estimation research on indigenous African faces and the 
fact that this work was experimented on two different ethnicities (Caucasian and Indigenous African) is a desirable feature in age estimation.

In future works, we hope to experiment the proposed approach with some other age ranking and estimation functions other than LSBoost upon our observation of it poor ranking performance, however, when ranks were accurately predicted, it estimates ages quite accurately. We also intend to extend this work by experimenting on a larger multi-ethnic dataset and a wider age range so that the generalization of the proposed approach across ethnicities could be improved upon. We also hope to experiment this approach with pruning in order to improve age estimation accuracy.

\section{ACKNOWLEDGMENT}

The authors wish to thank the Computer Science department, University of Ibadan and the International School Ibadan, Ibadan, Nigeria for granting permission to obtain the facial images of their students for the collation of our FAGE dataset and Professor Andreas Lanitis for providing us with the FG-NET dataset for this work.

\section{REFERENCES}

[1] J. T. Todd, L. S. Mark, R. E. Shaw, and J. B. Pittenger, "The Perception of Human Growth," Sci. Am., vol. 242, no. 2, pp. 132-144, 1980.

[2] Y. H. Kwon and V. Lobo, "Age Classification from Facial Images," Comput. Vis. Image Underst., vol. 74, no. 1, pp. $1-21,1999$.

[3] W. Chao, J. Liu, and J. Ding, "Facial Age Estimation based on Label-Sensitive Learning and Age-Oriented Regression," Pattern Recognit., vol. 46, no. 3, pp. 628641, 2013.

[4] A. M. Albert, K. Ricanek, and E. Patterson, "A Review of The Literature on The Aging Adult Skull and Face: Implications for Forensic Science Research and Applications," pp. 1-9, 2007.

[5] G. Guo and T. S. Huang, "Human Age Estimation Using Bio-Inspired Features," in IEEE Conference on Computer Vision and Pattern Recognition, 2009, pp. 112-119.

[6] X. Geng, Y. Fu, and K. S. Miles, "Automatic Facial Age Estimation," in 11th Pacific Rim International Conference on Artificial Intelligence, 2010, pp. 1-130.

[7] G. Guo, Y. Fu, C. R. Dyer, and T. S. Huang, "ImageBased Human Age Estimation by Manifold Learning and Locally Adjusted Robust Regression," IEEE Trans. Image Process., vol. 17, no. 7, pp. 1178-1188, 2008.

[8] O. F. W. Onifade and J. D. Akinyemi, "A Model of Correlated Ageing Pattern for Age Ranking," in Fourth International Conference on Computer Science and Information Technology, 2014, vol. 4, no. 2, pp. 477-485.

[9] N. Ramanathan, R. Chellappa, and S. Biswas, "Age Progression in Human Faces: A Survey," Vis. Lang. Comput., pp. 1-11, 2009.

[10] "FG-NET," 2013. [Online]. Available: http://sting.cycollege.ac.cy/ alanitis/fgnetaging/index.htm. [Accessed: 17-Jun-2013].

[11] K. Ricanek and T. Tesafaye, "MORPH: A Longitudinal Image Database of Normal Adult Age-Progression," in In IEEE 7th International Conference on Automatic Face and Gesture Recognition, 2006, pp. 341-345.

[12] M. Y. El Dib and H. M. Onsi, "Human Age Estimation
Framework Using Different Facial Parts," Egypt. Informatics J., vol. 12, no. 1, pp. 53-59, 2011.

[13] G. Guo and G. Mu, "Simultaneous Dimensionality Reduction and Human Age Estimation via Kernel Partial Least Squares Regression," in IEEE Computer Vision and Pattern Recognition (CVPR), 2011 ..., 2011, pp. 657-664.

[14] O. F. W. Onifade and S. O. Ademola, "Fuzzy Analysis and Adaptive Anthropometry Model for Object Identification in Surveillance System," Int. J. Comput. Sci. Its Appl., vol. 20, no. 2, pp. 29 - 37, 2013.

[15] W. Horng, C. Lee, and C. Chen, "Classification of Age Groups Based on Facial Features," Tamkang J. Sci. Eng., vol. 4, no. 3, pp. 183-192, 2001.

[16] N. Ramanathan and R. Chellappa, "Face Verification across Age Progression," IEEE Trans. Image Process., vol. 15, no. 11, pp. 3349 - 3361, 2006.

[17] X. Geng, Z. Zhou, and K. Smith-miles, "Automatic Age Estimation Based on Facial Aging Patterns," IEEE Trans. Image Process., vol. 29, no. 12, pp. 2234-2240, 2007.

[18] A. Lanitis, C. J. Taylor, and T. F. Cootes, "Towards Automatic Simulation of Aging Effects on Face Images," vol. 24, no. 4, pp. 442-455, 2002.

[19] M. Tsai, Y. Liao, and I. Lin, "Human Face Aging With Guided Prediction and Detail Synthesis," Multimed. Tools Appl., 2013.

[20] A. Lanitis, C. Draganova, and C. Christodoulou, "Comparing Different Classifiers for Automatic Age Estimation," IEEE Trans. Syst. Man, Cybern. Part B Cybern., vol. 34, no. 1, pp. 621-628, 2004.

[21] X. Zhuang, X. Zhou, M. Hasegawa-Johnson, and T. Huang, "Face Age Estimation Using Patch-Based Hidden Markov Model Supervectors," in $19^{\text {th }}$ International Conference on Pattern Recognition, 2008, pp. 1-4.

[22] V. N. Vapnik, Statistical Learning Theory. New York: John Wiley \& Sons Inc., 1998, pp. 1-740.

[23] V. N. Vapnik, "An Overview of Statistical Learning Theory," IEEE Trans. Neural Networks, vol. 10, no. 5, pp. 988-999, 1999.

[24] Y. Fu, Y. Xu, and T. S. Huang, "Estimating Human Age by Manifold Analysis of Face Pictures and Regression on Aaging Features," in IEEE Conference on Multimedia Exposition, 2007, pp. 1383-1386.

[25] Y. Fu and T. S. Huang, "Human Age Estimation With Regression on Discriminative Aging Manifold," IEEE Trans. Multimed., vol. 10, no. 4, pp. 578-584, 2008.

[26] S. Yan, X. Zhou, M. Hasegawa-johnson, and T. S. Huang, "Regression from Patch-Kernel," in IEEE Conference on Computer Vision and Pattern Recognition, 2008, pp. 1-8.

[27] S. Yan, H. Wang, T. S. Huang, Q. Yang, and X. Tang, "Ranking with Uncertain Labels," in IEEE International Conference on Multimedia and Expo, 2007, pp. 96-99.

[28] D. Cao, Z. Lei, Z. Zhang, J. Feng, and S. Z. Li, "Human Age Estimation Using Ranking SVM," in $7^{\text {th }}$ Chinese Conference, CCBR, 2012, vol. 7701, pp. 324-331.

[29] K. Chang, C. Chen, and Y. Hung, "A Ranking Approach for Human Age Estimation Based on Face," in International Conference on Pattern Recognition, 2010.

[30] P. Yang, L. Zhong, and D. Metaxas, "Ranking Model for Facial Age Estimation," in International Conference on Pattern Recognition, 2010, pp. 3408-3411.

[31] K. Chang, C. Chen, and Y. Hung, "Ordinal Hyperplanes Ranker with Cost Sensitivities for Age Estimation," in IEEE Conference on Computer Vision and Pattern Recognition, 2011, pp. 585 - 592.

[32] O. F. W. Onifade and J. D. Akinyemi, "A GW Ranking Approach for Facial Age Estimation," Egypt. Comput. Sci. J., vol. 38, no. 3, pp. 1-6, 2014. 
[33] Z. Cao, T. Qin, T.-Y. Liu, M.-F. Tsai, and H. Li, "Learning to Rank: From Pairwise Approach to Listwise Approach," in $24^{\text {th }}$ International Conference on Machine Learning, 2007, pp. 1-8.

[34] T. Qin, X.-D. Zhang, M.-F. Tsai, D.-S. Wang, T.-Y. Liu, and H. Li, "Query-level Loss Functions For Information Retrieval," Inf. Process. Manag., vol. 44, no. 2, pp. 838855, Mar. 2008.

[35] F. Xia and J. Wang, "Listwise Approach to Learning to Rank - Theory and Algorithm," in $25^{\text {th }}$ International Conference on Machine Learning, 2008.

[36] T. Ojala, M. Pietikäinen, and D. Harwood, "A Comparative Study of Texture Measures with Classification Based on Feature Distributions," Pattern Recognit. Lett., vol. 19, no. 3, pp. 51-59, 1996.

[37] T. Ojala, M. Pietikäinen, and T. Mäenpää, "Gray Scale and Rotation Invariant Texture Classification with Local Binary Patterns," in European Conference on Computer Vision, 2000, pp. 404-420.

[38] T. Ojala, M. Pietikäinen, and M. Topi, "Multiresolution Gray-Scale and Rotation Invariant Texture Classification with Local Binary Patterns," IEEE Trans. Pattern Anal. Mach. Intell., vol. 24, no. 7, pp. 971-987, 2002.

[39] G. Guo, T. S. Huang, and C. R. Dyer, "Locally Adjusted Robust Regression for Human Age Estimation," in IEEE Workshop on Applications of Computer Vision, 2008.

[40] T. Hastie and R. Tibshirani, The Elements Of Statistical Learning: Data Mining, Inference And Prediction, $2^{\text {nd }}$ ed. Springer, 2008, pp. 1-758.

[41] K. Luu, K. Ricanek, T. D. Bui, and C. Y. Suen, "Age Estimation using Active Appearance Models and Support Vector Machine Regression," in IEEE International Conference on Biometrics: Theory, Applications and System, 2009, pp. 314-318.

[42] M. Y. El Dib and M. El-saban, "Human Age Estimation
Using Enhanced Bio-Inspired Features (EBIF)," in IEEE $17^{\text {th }}$ International Conference on Image Processing, 2010, pp. 1589-1592.

\section{Authors' Profiles}

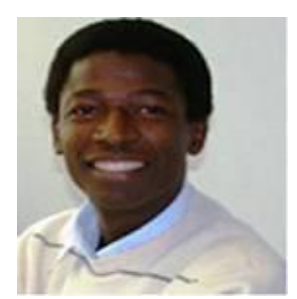

Olufade F. W. Onifade obtained a $\mathrm{PhD}$ in computer science from Nancy 2 University, Nancy, France in 2009. He is currently a Lecturer at the Computer Science department, University of Ibadan, Ibadan, Nigeria. $\mathrm{He}$ has published over 70 papers in both local and International referred journals and conferences and has held several fellowships including ETT-MIT and the CV Raman Fellowship for African Researchers in India. His research interests include Fuzzy Learning, Information Retrieval, Biometrics \& Pattern Matching. Dr. Onifade is a member of IEEE, IAENG and CPN.

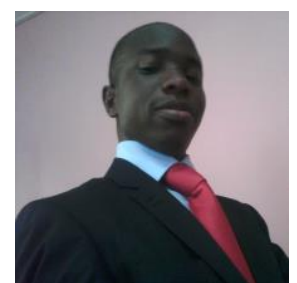

Damilola J. Akinyemi obtained his B.Sc. and M.Sc. degrees from University of Ilorin and University of Ibadan, Nigeria in 2010 and 2014 respectively.

His research interests include Computer Vision, Image Processing and Pattern Recognition.

How to cite this paper: Olufade F. W. Onifade, Damilola J. Akinyemi,"GWAgeER - A GroupWise Age Ranking Framework for Human Age Estimation", IJIGSP, vol.7, no.5, pp.1-12, 2015.DOI: 10.5815/ijigsp.2015.05.01 